\title{
Novel mutations of TYK2 leading to divergent clinical phenotypes
}

\author{
Ge Lv ${ }^{1}$, Gan Sun ${ }^{1}$, Peilin $\mathrm{Wu}^{2}$, Xiao Du ${ }^{1}$, Ting Zeng ${ }^{1}$, Wen Wen ${ }^{1}$, Lina Zhou ${ }^{1}$, Yun-Fei \\ $\mathrm{An}^{3}$, Xue-Mei Tang ${ }^{4}$, Tingyan $\mathrm{He}^{5}$, Xiao-dong Zhao ${ }^{1}$, and Hongqiang $\mathrm{Du}^{1}$ \\ ${ }^{1}$ Chongqing Medical University Affiliated Children's Hospital \\ ${ }^{2}$ Fujian Medical University Affiliated Fuzhou First Hospital \\ ${ }^{3}$ Children's Hospital of Chongqing Medical University, Ministry of Education Key \\ Laboratory of Child Development and Disorders \\ ${ }^{4}$ Children's Hospital, Chongqing Medical University \\ ${ }^{5}$ Shenzhen Children's Hospital
}

May 28, 2021

\begin{abstract}
Background: TYK2 deficiency is a rare Primary immunodeficiency disease caused by loss of function mutations of TYK2 gene, which is initially proposed as a subset of Hyper IgE syndrome (HIES). However, accumulating evidence suggest TYK2 deficient patients do not necessarily present with HIES characteristics, indicating a vacuum of knowledge on the exact roles of TYK2 in human immune system. Method: Pathogenic effects of patients were confirmed by qRT-PCR, western blot and protein stability assays. The responses to cytokines including IFN- $\alpha / \beta / \gamma$, IL-6, IL-10, IL12 and IL-23 of peripheral blood mononuclear cells (PBMCs) from these patients were detected by western blot, qRT-PCR and flow cytometry. The differentiation of T and B cells were detected by flow cytometry. Results: We describe five more TYK2 deficient cases presenting with or without hyper IgE levels, atopy and distinct pathogen infection profile, which are caused by novel TYK2 mutations. These mutations were all found by high throughout sequencing and confirmed by Sanger sequencing. The patients showed heterogenous responses to various cytokine treatments, including IFN- $\alpha / \beta / \gamma$, IL-6, IL-10, IL12 and IL-23. The homeostasis of lymphocytes is also disrupted. Conclusion: Based on our findings, we propose that TYK2 works as a multi-tasker in orchestrating various cytokines signaling pathways, differentially combined defects of which account for the expressed clinical manifestations.
\end{abstract}

\section{Introduction}

The non-receptor tyrosine kinase 2 (TYK2) is a member of the Janus kinase (JAK) family consisting of three additional members (JAK1-3). Cytokine binding to respective receptor complexes (type I or type II) activates JAKs, which subsequently phosphorylate intracellular receptor chain residues and activate a family of transcription factors termed signal transducers and activators of transcription (STATs, comprised of STAT1-4, STAT5A, STAT5B and STAT6) [1].

Within the JAK-STAT family of proteins, several primary immunodeficiencies have been described, ranging from autosomal recessive severe combined immunodeficiency (AR-SCID) in the case of JAK3 mutations, over a moderately severe phenotype in the case of autosomal dominant Hyper-IgE syndrome (AD-HIES) involving STAT3 mutations, into a heterogeneous clinical presentation in STAT1 deficiency with autosomal recessive Mendelian susceptibility to mycobacterial disease (AR-MSMD), and finally to a relatively mild phenotype in the case of TYK2 deficiency ${ }^{[2]}$.

To date, there are 13 cases in total in English literature ${ }^{[3-8]}$. The first TYK2-deficiency patient was reported in 2006, a 22-years-old male Japanese, who displayed BCG lymphadenitis, $S$. aureus infections and recurrent viral infection, which were attributed to almost abolished responses of patient's cells to IL-23, IL-12 and 
type I interferon (type I IFN) treatment, respectively [3]. Most interestingly, he also presented with the triad of signs characteristic of HIES: atopic dermatitis, high circulating IgE levels, and recurrent cutaneous staphylococcal infections, which led to the proposal that TYK2-deficiency should be a subset of AR-HIES. However, this notion was challenged lately by a comprehensive study of seven TYK2-deficiency patients showing normal IgE levels and absent of atopic dermatitis or cutaneous staphylococcal infection [4]. Later on, both HIES-like and non-HIES TYK2-deficiency cases were reported ${ }^{[5-8]}$ and more confusingly, it has been shown identical TYK2 mutation can result in both HIES-like and non-HIES phenotypes ${ }^{[5,7]}$. Thus, more cases and further investigations are required to fully understand the nature of TYK2-deficiency.

Here, we present five cases of TYK2-deficiency with novel mutations from five unrelated Chinese families. Briefly, a 2+-yr-old boy (P1) suffered from repeated pneumonia, stomatitis, perilabial herpes and thrush since the age of 3 months; a 3-yrs-old boy (P2) suffered from recurrent respiratory tract infections and diarrhea since the age of 6 months; a 10-yrs-old girl (P3) suffered from recurrent respiratory infection since 2 years old and she had a history of refractory eczema; a 5-yrs-old boy (P4) suffered from recurrent suppurative otitis media and severe pneumonia and he also had a history of eczema and was highly allergic; a 5-yrs-old boy (P5) suffered from recurrent pneumonia and refractory eczema and he also displayed hypothyroidism. By investigating these cases and comparing with previously reported cases, we aim to uncover a more complete picture of TYK2 deficiency.

\section{Methods}

\section{Patients}

The ethics committee of Children's Hospital of Chongqing Medical University approved the study. Written informed consent for participation in the study were obtained from patients' parents. Blood samples from patients and unaffected relatives were collected for molecular studies, which were performed in accordance with the Declaration of Helsinki.

\section{Genetic Analyses}

Genomic DNA were isolated from peripheral blood samples. Disease-causing mutations were screened using whole genome sequencing (WES) or targeted next generation sequencing (NGS) (MyGenostics, Inc. and Chigene, Inc.). Candidate mutations were confirmed by Sanger sequencing (primer sequences were listed Supplementary table 1).

\section{Western blotting}

PBMCs were stimulated with various cytokines for indicated time. The extracted protein was subjected to 10\% SDS-PAGE and transferred onto PVDF membranes (Millipore). The following primary antibodies were used: rabbit anti-phosphorylated STAT1 (Cell Signaling Technology) and rabbit anti-STAT1 (Cell Signaling Technology), rabbit anti-phosphorylated STAT3 (Cell Signaling Technology), mouse anti-STAT3 (BD), rabbit anti-TYK2(Abcam, cat223733), and that against $\beta$-actin rabbit mAb horseradish peroxidase (HRP)conjugated (Cell Signaling Technology). The HRP-conjugated goat anti-rabbit IgG secondary antibody (Cell Signaling Technology) and the HRP-conjugated goat anti-mouse IgG secondary antibody (ZSGB-BIO, China).

\section{Flow Cytometry Assay}

The following antibodies were used: CD3-PerCP (HIT3a), CD4-FITC (RPA-T4), CD8-BV510 ( RPA-

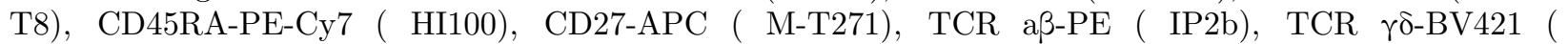
B1), CD19-APC ( HIB19), CD27-V450 ( M-T271), IgD-AF488 ( IA6-2), CD24-PE ( ML5), and CD38-PerCP ( HIT2), CD4-PE-Cy7 ( RPA-T4), CXCR5-BV421 ( J25ID4), CD25-APC ( MT271, ), CD127-PE ( A019D5, ), CCR6-PE ( G034E3, ), CD25-BV421 ( BC96, ), Helios-PerCP-cy5.5 ( 22F6, ), CD19-PerCP-Cy5.5 ( SJ25C1, ), CD27- PE-Cy7 ( MT271). All were from Biolegend. CD45RO-APC (UCHL 1), CD45RA-FITC (HI100), CXCR3-APC (1C6), and FOXP3-PE (PCH101), CD152-APC (BNI3), IgM-APC (G20-127) and IFN- $\gamma-A P C$ (4S.B3). All were from 
BD Bioscicences. Th1 was defined as $\mathrm{CD}^{+}{ }^{+} \mathrm{CD} 45 \mathrm{RA}^{-} \mathrm{CXCR} 5^{-} \mathrm{CXCR} 3^{+} \mathrm{CCR} 6{ }^{-}$, Th2 was defined as CD $4{ }^{+}$CD 45RA ${ }^{-}$CXCR5 ${ }^{-}$CXCR3 ${ }^{-}$CCR6 ${ }^{-}$, Th17 cells CD4 ${ }^{+}{ }^{-}$D 45 RA $^{-}$CXCR5 ${ }^{-}$CXCR3 ${ }^{-}$CCR6 ${ }^{+}$, Th1/17 cells $\mathrm{CD} 4{ }^{+} \mathrm{CD} 45 \mathrm{RA}^{-}{ }^{-} \mathrm{XCR} 5{ }^{-} \mathrm{CXCR} 3{ }^{+} \mathrm{CCR} 6+$, Th1-like cells $\mathrm{CD} 4^{+} \mathrm{CD} 45 \mathrm{RA}^{-} \mathrm{CXCR} 5^{+} \mathrm{CXCR} 3^{+} \mathrm{CCR} 6{ }^{-}$, Th2like cells $\mathrm{CD}^{+}{ }^{+} \mathrm{CD} 45 \mathrm{RA}^{-} \mathrm{CXCR}{ }^{-} \mathrm{CXCR}^{-} \mathrm{CCR} 6^{-}$, Th17-like cells $\mathrm{CD} 4^{+} \mathrm{CD} 45^{-}{ }^{-} \mathrm{CXRR}^{+}{ }^{+} \mathrm{CXCR} 3^{-} \mathrm{CCR} 6^{+}$, Th1/17-like cells $\mathrm{CD} 4{ }^{+} \mathrm{CD} 45 \mathrm{RA}^{-} \mathrm{CXCR} 5^{+} \mathrm{CXCR}^{+} \mathrm{CCR} 6+, \quad \mathrm{T}$ follicular helper cell $\mathrm{CD}^{+}{ }^{\mathrm{CD}} 4^{+} \mathrm{CD}_{4} 5 \mathrm{RA}^{-} \mathrm{CXCR}^{+}$, circulating follicular regulatory $\mathrm{T} \mathrm{CD}^{+} \mathrm{CD}^{+} \mathrm{CD} 45 \mathrm{RA}^{+} \mathrm{CXCR}^{+}$, Treg $\mathrm{CD}^{+}{ }^{+} \mathrm{CD} 4^{+} \mathrm{CD} 25^{-} \mathrm{FOXP}^{+}, \mathrm{IgM}^{\mathrm{hi}} \mathrm{B}$ cells $\left(\mathrm{CD} 19^{+} \mathrm{CD} 27^{-} \mathrm{IgM}^{+}\right)$, MZ-like B $\left(\mathrm{CD}^{-} 9^{+} \mathrm{CD} 27^{+} \mathrm{IgM}^{+}\right)$, switched-memory B $\left(\mathrm{CD} 19^{+} \mathrm{CD} 27^{+} \mathrm{IgM}^{-}\right)$.

The intracellular production of IFN- $\gamma$ was investigated in PBMCs by flow cytometry. PBMCs $\left(2 \times 10^{6}\right.$ cells $/ \mathrm{ml})$ were either unstimulated or stimulated with PMA $(50 \mathrm{ng} / \mathrm{ml})$ and Ionomycin $(500 \mathrm{ng} / \mathrm{ml})$ for 5 hrs or BCG (MOI = 20) or $100 \mathrm{ng} / \mathrm{ml} \mathrm{BCG} \mathrm{+} \mathrm{IL-12} \mathrm{for} 72 \mathrm{hrs,} \mathrm{in} \mathrm{24-well} \mathrm{plates.} \mathrm{All} \mathrm{the} \mathrm{samples} \mathrm{were} \mathrm{treated}$ with $1 \mu \mathrm{g} / \mathrm{ml}$ GolgiPlug (BD) for the last 2 or $6 \mathrm{hrs}$ of culture.

The staining was performed according to manufacturers' guides. The samples were acquired on a FACSCanto II flow cytometer, and the data were analyzed using FlowJo.

\section{Real-Time Quantitative PCR}

Total RNA was extracted from peripheral blood with the Blood Total RNA Miniprep Kit (Axygen). The cDNA was transcribed by the Transcriptor cDNA Synth. Kit (Roche). Levels of targeted mRNA were determined using a Bio-rad CFX96 Touch (BIO-RAD) machine. The results of each gene were determined with the 2- $\Delta \Delta \mathrm{Ct}$ method, and data was expressed as fold induction. Primers were listed in Supplementary table 2 .

\section{Construction of plasmids}

Full length human TYK2 cDNA template (NM003331) was purchased from YouBio (G114136), Shanghai China and subcloned to 7.1-3 $\times$ Flag-CMV expression plasmid as wild type TYK2 (WT) using Seamless Cloning kit from Biomed (CL116), Beijing China. Various missense and nonsense mutation mutants of TYK2 were generated by PCR method using WT as template. Mutations were confirmed by Sanger sequencing.

\section{Cycloheximide Chase Assay}

This assay is adapted from previous report ${ }^{[9]}$. Briefly, HEK293T cells were plated and transfected with various WT and mutant expression plasmids. 24 hours later, cycloheximide was added $(1 \mathrm{ug} / \mathrm{ml})$ and cells were collected at different time point for western blot detection.

\section{Statistical analysis}

Samples were compared using two-tailed, unpaired Student's $t$-test with GraphPad Prism 7.00 if applicable, unless otherwise stated. Error bars were represented by SEM. ${ }^{*} P<0.05$, ${ }^{* *} P<0.01,{ }^{* * *} P<0.001$, ${ }^{* * * *} P<$ 0.0001 .

\section{Results}

\section{Clinical courses strongly favor a diagnosis of PID (Table 1).}

Patient 1 is a 2-yrs-old boy who suffered from perianal abscess at 26 days and repeated pneumonia, stomatitis (SupFig. 1A), perilabial herpes (SupFig. 1B) and thrush since the age of 3 months. In addition, he also developed suppurative meningitis with unknown pathogen at 9 months old, salmonella enteritidis and handfoot-and-mouth disease at 1 year old. He had a history of eczema and was allergic to pollen. He had received the following vaccinations:Bacille Calmette-Guerin (BCG),Hepatitis B, Polio, Diphtheria-pertussis-tetanus (DPT). BCG vaccination site was inflamed and purulent, and scab was formed after 9 months (SupFig. 1C). Laboratory findings included mild elevated $\mathrm{IgE}$ level $(330 \mathrm{IU} / \mathrm{mL}, 516.3 \mathrm{IU} / \mathrm{mL})$ and positive viremia of EBV, CMV and HSV-1. 
Patient 2 is a 3-yrs-old boy who suffered from recurrent respiratory tract infections and diarrhea since the age of 6 months. Pathogens found during multiple hospitalizations included Salmonella, Mycoplasma pneumonia , and Mycobacterium Bovis BCG. No signs of viral or fungal infections were noticed. No high serum IgE concentration, atopy, staphylococcal illness, or lymphopenia were found. Additionally, he also had language delay and was diagnosed of autism at the age of 3. Detailed clinical manifestations of this patient have been published elsewhere recently ${ }^{[8]}$.

Patient 3 is a 10-yrs-old girl who suffered from recurrent respiratory infection with unknown pathogens since she was 2 years old. She was vaccinated with hepatitis B, BCG, Polio, DPT and measles vaccines and showed no abnormal responses. She had a history of refractory eczema. Laboratory findings included low levels of immunoglobulin:IgG 1.55g/L, IgA $0.0667 \mathrm{~g} / \mathrm{L}, \operatorname{IgM} 0.172 \mathrm{~g} / \mathrm{L}, \operatorname{IgE} 0.6 \mathrm{IU} / \mathrm{mL}$, increased eosinophils and negative evidence of tubercle bacilli .

Patient 4 is a 5 -yrs-old boy who suffered from recurrent suppurative otitis media and severe pneumonia. He was vaccinated with BCG and hepatitis B vaccine and there was pus at the BCG inoculation site. Pathogens found during multiple hospitalizations includedStaphylococcus haemolyticus, Staphylococcus epidermidis , Mycoplasma pneumoniae and HSV-II. He had a history of eczema and was highly allergic. Laboratory findings included high level of $\operatorname{IgE}\left(756.83^{\sim} 1392 \mathrm{IU} / \mathrm{mL}\right)$ and increased eosinophils.

Patient 5 was a 5 -yrs-old boy who suffered from recurrent pneumonia with unknown pathogens and refractory eczema. BCG was injected immediately after birth and he also developed BCG-itis (SupFig. 1D). Laboratory findings included high level of $\mathrm{IgE}\left(1325^{\sim} 2030 \mathrm{IU} / \mathrm{mL}\right)$, increased eosinophils and negative evidence of TB infection. The patient also displayed secondary hypothyroidism: T3 $1.55 \mathrm{nmol} / \mathrm{L}, \mathrm{FT} 33.71 \mathrm{pmol} / \mathrm{L}, \mathrm{FT} 4$ $11.53 \mathrm{pmol} / \mathrm{L}$, TPOAb $177.1 \mathrm{U} / \mathrm{mL}$.

All patients were born to non-consanguineous and healthy parents. They were all clinically diagnosed with PID.

\section{Novel mutations identified by WES or targeted NGS lead to TYK2 deficiency}

The mutations were identified by whole exome sequencing (WES) for P1, P3 and P5 or targeted next generation sequencing (NGS, PID gene panel) for P2 and P4 (Fig. 1A-1C). P1 carried novel compound heterozygous mutations, c.3041T >C, p.L1014P (MAF: EXAC 0.000008; CADD score 32, pathogenic) and c.1253C >A, p.S418X (pathogenic) in TYK2 (Fig. 1D). P2 carried a novel homozygous mutation, c.2395G >A, p.G799R (MAF: EXAC 0.000008; CADD score 28.5, pathogenic) in TYK2 (Fig. 1D). P3 carried novel compound heterozygous mutations, c.1621G >A, p.G541R (MAF: EXAC 0.000058; CADD score 23, pathogenic) and c.704G>A, p.R235Q (MAF: EXAC 0.000114653; CADD score 22.4, pathogenic) in TYK2 (Fig. 1D). P4 carried compound heterozygous mutation, c.2269C $>$ G, p.L757V (MAF: no recorded; CADD score 33, pathogenic) and c.149delC p. S50CfsX2 (pathogenic) in TYK2 (Fig. 1D). P5 carried a novel homozygous mutation, c.1507C > T, p.R503X (pathogenic) in TYK2 (Fig. 1D). These mutations were all further confirmed by Sanger sequencing (Fig. 1D). According to inhouse prediction algorithm developed by the sequencing companies, these mutations were all predicted to be pathogenic to the expression or function of TYK2 protein (Fig. 1B and 1C). To confirm this, we then determined the protein level of TYK2 in patients' PBMCs and we found almost abolished expression of TYK2 (Fig. 1E-1H) in all patients except for P4, whose blood sample was not available. The anti-TYK2 antibody recognizes 200-400 amino acids of TYK2. We also determined the mRNA level of TYK2 in P1, P2 and P3, we found that their mRNA levels were decreased (Fig. 1I-1K).

To further understand and confirm the pathogenicity of these mutations, we performed Cycloheximide Chase Assay to test the protein stability. We found that these mutations indeed impaired the protein stability (SupFig. 2). Please note that mutation in P2 has been tested to impaired the protein stability previously ${ }^{[8]}$. Taken together, these five patients from five unrelated families all had TYK2 deficiency.

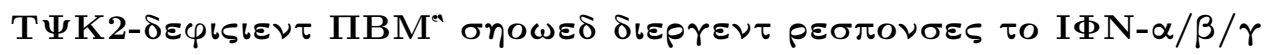

Type I IFNs signal through IFNAR1 and IFNAR2, which associate with TYK2 and JAK1, respectively. 
STAT1 and STAT2 are the major signal transducing STATs in response to type I IFNs ${ }^{[10-12]}$. Nevertheless, also all other STATs can be activated and contribute to responses in a cell type-specific manner. IFN- $\gamma$ signals through IFNGR1 and IFNGR2, which are associated with JAK1 and JAK2, respectively ${ }^{[13]}$ and then STAT1 is activated. Often STAT3 ${ }^{[14,15]}$ and sometimes STAT5 ${ }^{[16]}$ are also activated by IFN- $\gamma$. To further investigate the functional effects of TYK2 deficiency, we tested the responses of PBMC from TYK2-deficient patients to type I and type II interferons. For P1, the phosphorylation of STAT1 and STAT3 induced by IFN$\alpha$ and IFN- $\beta$ were both abolished, while the phosphorylation of STAT1 induced by IFN- $\gamma$ was comparable with that of healthy control (Fig. 2A). Reduced STAT1 expression is believed to be partially responsible for reduced phosphorylation of STAT1 in response to type I interferons in TYK2 deficient cells. Consistently, RT-qPCR showed decreased transcription of various ISGs (Fig. 2C). P2 also showed impaired responses to IFN- $\alpha / \beta$ but normal response to IFN- $\gamma$ (Fig. 2B), which was also consistent with ISG expression (Fig. 2D). Taken together, our data indicates that TYK2 deficiency results in divergent responses to IFN- $\alpha / \beta / \gamma$.

\section{TYK2-deficient PBMC showed divergent responses to IL-10 and IL-6}

IL-10 signals via IL-10R1 and IL-10R2, which associate with JAK1 and TYK2, respectively [17]. IL-6 can also induce phosphorylation of TYK2. Similarly, we assessed the responses of PBMC from TYK2-deficient patients to IL-10 and IL-6. PBMC from P1 showed intact response to IL-6 but abolished response to IL-10 as evidenced by the phosphorylation of STAT3 (Fig. 3A). PBMC from P2 showed significantly impaired responses to both IL-6 and IL-10 as evidenced by the phosphorylation of STAT3 (Fig. 3B), in contrary to P3, whose responses to IL-10 and IL-6 seemed normal as evidenced by the phosphorylation of STAT3 (Fig. 3C) and the transcription of SOCS3 (Fig. 3D), a STAT3 target gene. These data again showed the role of TYK2 in IL-10 and IL-6 signaling was complicated as previously reported.

\section{TYK2-deficient PBMC showed similar responses to IL-12 and IL-23}

TYK2 associates with IL-12R $\beta 1$, a receptor chain that is shared by IL-12 and IL-23. The second receptor chain for both cytokines (IL-12R $\beta 2$ and IL-23R, respectively) associates with JAK2. Biological responses are mainly mediated by STAT4 in response to IL-12 and STAT3 in response to IL-23 [17]. We then assessed the responses of PBMC from TYK2-deficient patients to IL-12 and IL-23. In contrast to the responses to IL-6 and IL-10, PBMC from P2 and P3 showed similar impairment in response to IL-12 and IL-23 treatment (Fig. 4A-4D). Consistently, IFN- $\gamma$ production by T cells from P3 was also less compared to healthy control when treated with BCG alone or BCG plus IL-12 (Fig. 4E). Collectively, these data shown so far indicate TYK2 deficiency can lead to divergent clinical phenotypes possibly due to divergent signaling defects.

\section{TYK2 deficiency disturbs homeostasis of lymphocytes}

Cytokines signaling plays essential roles in controlling homeostasis of immune system [18]. The impairment of various cytokines signaling in TYK2 deficient patients could lead to disruption of this balance. P1 had decreased $\mathrm{CD}^{+}{ }^{+} \mathrm{T}$ cells, especially the CD4 naïve cells, while his CD4 CM (central memory) and CD4 EM (effect memory) cells were increased. Besides, total B cells of P1 was degressive with elevated naïve B cells and transitional B cells (Supplementary Table 3). In P2, except for the decline of NK cells, the subsets of T cells and B cells were normal (Supplementary Table 3). Although the subsets of T cells in P3 were normal, B cells were significantly different from those in normal subjects. The total B cells, memory B cells and plasmablasts B cells were declined but the naïve B cells and the transitional B cells were higher than normal range (Supplementary Table 3). Similar to P1, P5 had abnormal T cells, the total T cells, CD4 ${ }^{+} \mathrm{T}$ cells, $\mathrm{CD}^{+} \mathrm{T}$ cells were significantly decreased, B cells and NK cells were normal (Supplementary Table 3).

Further analysis of CD4 ${ }^{+} \mathrm{T}$ cell subsets showed that frequencies of Th1 and Th1-like cells were increased in P1 and P2 (Fig. 5A-5F), while frequency of Th2 cells was decreased in P1, P2 and P3 compared to healthy controls (Fig. 5A-5F). Th17 cells seemed unaffected in all three patients tested. The frequencies of Tfh cells were elevated in P1 and P3, but normal in P2 (Fig. 5G). We further analyzed the Tfh subsets showing normal Tfr frequency and increased CXCR $5^{+} \mathrm{PD}-1^{+}$cells (Fig. $5 \mathrm{H}-5 \mathrm{~L}$ and $5 \mathrm{~N}$ ). The proportion of Treg were comparable to that of healthy controls in P2 and P3, but increased in P3 (Fig. 5H-5L). 
For B cell compartment, IgM ${ }^{\text {hi }}$ B cells were both elevated in P2 and P3. MZ-like cells and Sm-B cells were normal in P2, but decreased in P3 (Fig. 5M and 5O-P).

\section{Discussion}

Clinical primary immunodeficiency cases caused by TYK2 mutations are very rarely reported. To our best knowledge, there are 13 cases in total in English literature not including ours. Because of this, the exact roles of TYK2 in the development, function and regulation of immune cells in human remain largely unknown. In clinical setting, it is extremely difficult to raise a specific suspicion of TYK2 deficiency when there is no clear full picture of its clinical manifestations which are closely related to the roles TYK2 plays in various immunologic processes. A recent study showed the P1104A TYK2 common variant predisposes host to mycobacteria infection by selectively disrupting IL-23-dependent antimycobacterial IFN- $\gamma$ immunity, which also contributed to our understanding of TYK2 function ${ }^{[19]}$. In this study, we presented five more cases of TYK2 deficiency and investigated many aspects of the effects TYK2 deficiency brings, which we think would further improve our understanding of TYK2 deficiency.

The first TYK2-deficient patient to be described was Japanese and had HIES as the main clinical feature, associated with numerous intracellular infections. Since then, TYK2 deficiency was considered a subset of AR-HIES. However, newly identified TYK2 deficient patients did not necessarily present with hyper-IgE. A comprehensive comparison of various immunologic defects between the first reported patient and seven patients without hyper-IgE was reported, which proposed that intact IL-6/STAT3 signaling in patients without hyper-IgE might be responsible for this phenotypic difference ${ }^{[4]}$. As defects in STAT3-mediated signaling play a key role in the development of HIES ${ }^{[20]}$ and IL-6 signaling is TYK2 independent in mice ${ }^{[21]}$. Another TYK2 deficient patient without HIES was also reported to show normal IL-6 signaling ${ }^{[6]}$ and a TYK2 deficient patient with HIES showing impaired IL-6 signaling was reported ${ }^{[5]}$ (Table 1). Recently, autosomal recessive and dominant mutations in $I L 6 S T$, which encodes gp130, a subunit of cytokine receptor for IL-6, IL-11, IL-27 and some others, have been reported in patients with HIES ${ }^{[22,23]}$. Thus, this idea seems to be plausible according to these previously reported cases. Surprisingly, however, our data did not support this hypothesis. As shown in Fig. 3, PBMC from P1 with mildly elevated IgE and P3 with low IgE both responded to IL- 6 treatment normally in terms of STAT3 activation. Furthermore, PBMC from P2 with normal IgE level showed impaired response to IL-6 treatment. These findings strongly suggest the relation between hyper-IgE and TYK2 deficiency cannot be explained by dysregulated IL-6/STAT3 signaling.

Actually, mechanisms underlying increased IgE production are variable and complicated ${ }^{[24]}$ : a) enhanced class switching to IgE and the Th2 bias. B cell class switching to $\operatorname{IgE}$ is tightly regulated by interaction with CD4 T cells and the cytokine microenvironment. It is well established that IL-4 and IL-13, cytokines produced by Th2 cells and B cells, promote isotype class switching to IgE in B cells. Meanwhile, IL-10 and IL-21 act directly upon activated B cells to reduce the efficiency of class switching towards IgE. In addition, the engagement of CD40 on B cells by CD40L provides a second signal for IgE class switching [25]. Th bias towards a Th2 phenotype can indirectly promote a cytokine milieu capable of enhancing IgE production. b) defects in general tolerance, meaning the Th2 response is increased concurrently with Th1 and Th17 responses in cases of cataclysmic tolerance failure, which is seen in IPEX ${ }^{[26]}$. Intriguingly, P1, P2 and P3 in our cohort all showed Th1 bias instead, with variable IgE levels, indicating involvement of other mechanisms. c) defects in barrier. While the majority of patients with atopy begin with elevated IgE, a minority of patients develop atopic dermatitis with normal IgE levels but experience elevated IgE after prolonged disease, indicating increased IgE levels can be a result of disrupted barriers. So, it seems that in the case of TYK2 deficiency, disrupted cytokines signaling network is key to the normal, increased or decreased $\operatorname{IgE}$ levels.

The viral infections can probably be accounted for by defects of IFN- $\alpha / \beta / \gamma$ signaling. Similar to the first described TYK2 deficient patient, P1 in our cohort suffered repeated viral infection possibly due to abolished responses to IFN $-\alpha / \beta$. Although impaired but not abolished IFN signaling does not necessarily lead to severe viral infection as the case of P2 (Table 1 ) and previously reported cases ${ }^{[4]}$. The explanation for this might be that TYK2 plays important roles in various immune cells. Mutation of P2 may affected innate immune 
cells but not adaptive immune cells such as B cells, which also play critical role in restricting viral infection.

IL-12 signaling induced IFN- $\gamma$ production is believed to be responsible for the susceptibility to intracellular bacterial infection in TYK2 deficient patients, while IL-23 signaling induced IL-17 production for extracellular bacterial infection ${ }^{[3]}$. However, again our data present challenges to this notion as P3 showed no evidence of intracellular bacterial infection such as mycobacteria and salmonella in spite of impaired IL-12 signaling and P2 and P3 showed no evidence of extracellular bacterial infection such as S. aureusin spite of impaired IL-23 signaling. Similar to diverse responses to interferons, restricting extracellular or intracellular bacteria can be achieved by multiple cellular mechanisms.

Cytokine signaling not only affects the function of various immune cells but also plays essential roles in regulating homeostasis of immune system ${ }^{[18]}$. The effects of TYK2 deficiency on the homeostasis of lymphocytes compartment is unclear. In investigating this, we noticed that patients with elevated $\operatorname{IgE}(\mathrm{P} 1, \mathrm{P} 4$ and P5) levels shared similar profile of disrupted $\mathrm{T}$ and $\mathrm{B}$ cell compartment and patient with normal IgE level (P2) shared similar profile of $\mathrm{T}$ cell compartment but distinct profile of $\mathrm{B}$ cell compartment with patient with low IgE level (P3).

The divergent cellular responses to cytokines, which is probably responsible for the divergent clinical phenotypes, might be resulted from residual expression of mutant TYK2. Another possibility is that the presence/absence of the cell population that should respond caused these divergent cellular responses as the experiments in our study are done on heterogeneous cell populations. The effects of these mutations on TYK2 protein stability could be due to disrupted post-translation modification of the protein.

In summary, we presented five more TYK2 deficiency cases caused by different novel mutations displaying divergent cellular defects and variable clinical phenotypes, which demonstrated that the correlation of cellular defects and clinical phenotypes is far more complicated than previously thought. In view of this, here we propose a new model that TYK2 works as a multi-tasker in orchestrating various cytokines signaling pathways, differentially combined defects of which account for the expressed clinical manifestations. Furthermore, we speculated that the discrepancies in cellular defects could at least partially be explained by different mutational effects to TYK2 protein, which needs to be investigated in the future. Nevertheless, our findings might lead us further towards more accurate understanding of TYK2 function in human immune system and a more timely diagnosis of TYK2 deficient patients.

\section{ACKNOWLEGEMENT}

We are grateful to all the patients and their families for their continuous corporation in this study. We thank the members of the laboratory for their technical assistance. We thank doctors and nurses for their generous supporting this project.

\section{Impact Statement}

TYK2 deficiency is a rare primary immunodeficiency disease caused by loss of function mutations of TYK2 gene. Due to rarely reported cases, the nature of TYK2 deficiency and the function of TYK2 in human immune system are poorly understood. This study describes five TYK2 deficient cases presenting with or without hyper IgE levels, atopy and distinct pathogen infection profiles, which are caused by novel TYK2 mutations. Peripheral blood mononulear cells (PBMCs) from these patients showed heterogenous responses to various cytokines treatment, including IFN- $\alpha / \beta / \gamma$, IL-6, IL-10, IL12 and IL-23. The homeostasis of lymphocytes is also disrupted. Based on our findings, we propose that TYK2 works as a multi-tasker in orchestrating various cytokines signaling pathways, differentially combined defects of which account for the expressed clinical manifestations.

\section{ETHICS STATEMENT}

The ethics committee of Children's Hospital of Chongqing Medical University approved the study.

Blood samples collection were performed in accordance with the Declaration of Helsinki. 


\section{References}

1. Villarino, A.V., Kanno, Y., O'Shea, J.J. Mechanisms and consequences of Jak-STATsignaling in the immune system. Nature Immunology (2017) 18(4):374-384.

2. Casanova, J. L., Holland, S.and Notarangelo, L.. Inborn Errors of Human JAKs and STATs. Immunity (2012) 36(4):515-528.

3. Minegishi, Y., Saito, M., Morio, T., Watanabe, K., Agematsu, K. and Tsuchiya, S., et al. Human Tyrosine Kinase 2 Deficiency Reveals Its Requisite Roles in Multiple Cytokine Signals Involved in Innate and Acquired Immunity. Immunity (2006) 25(5):745-755.

4. Kreins, A. Y., Ciancanelli, M. J., Okada, S., Kong, X. F., N Ramírez-Alejo, and Kilic, S. S., et al. Human TYK2 deficiency: Mycobacterial and viral infections without hyper-IgE syndrome. Journal of Experimental Medicine (2015) 212(10):1641-1662.

5. Fuchs, S., Kaiser-Labusch, P., Bank, J., Ammann, S., Kolb-Kokocinski, A., and Edelbusch, C., et al. Tyrosine kinase 2 is not limiting human antiviral type III interferon responses. European Journal of Immunology (2016) 46(11):2639-2649.

6. Nemoto, M., Hattori, H., Maeda, N., Akita, N., Muramatsu, H. and Moritani, S., et al. Compound heterozygous TYK2 mutations underlie primary immunodeficiency with T-cell lymphopenia. Scientific Reports (2018) 8(1):6956-6968.

7. Sarrafzadeh, S.A., Mahloojirad, M., Casanova, J.L., Badalzadeh, M., Bustamante, J., Boisson-Dupuis, S., et al. A New Patient with Inherited TYK2 Deficiency. Journal of Clinical Immunology (2019) 40(1):232-235.

8. Wu, P., Chen, S., Wu, B., Chen, J. and Lv, G.. A TYK2 Gene Mutation c.2395G>A Leads to TYK2 Deficiency: A Case Report and Literature Review. Frontiers in Pediatrics (2020) 8,253.

9. Kao, S. H., Wang, W. L., Chen, C. Y., Chang, Y. L. and Yang, P. C.. Analysis of Protein Stability by the Cycloheximide Chase Assay.BIO-PROTOCOL (2015) 5(1):1-5.

10. Chen, X., Xu, Q., Li, X., Wang, L. and Zhao, X. . Molecular and Phenotypic Characterization of Nine Patients with STAT1 GOF Mutations in China. Journal of Clinical Immunology (2020) 40(1):82-95.

11. J Darnell, J., Kerr, I. and Stark, G.. Jak-STAT pathways and transcriptional activation in response to IFNs and other extracellular signaling proteins. Science (1994) 264(5164):1415-1421.

12. Schindler, C., and Plumlee, C.. Interferons pen the JAK-STAT pathway. Seminars in Cell \& Developmental Biology (2008) 19(4):311-318.

13. Pestka, S., Krause, C. D., and Walter, M.R. Interferons, interferon-like cytokines, and their receptors. Immunological Reviews (2010) 202(1):8-32.

14. Sattler, M., Durstin, M. A., Frank, D. A., Okuda, K., Kaushansky, K. and Salgia, R., et al. The thrombopoietin receptor c-MPL activates JAK2 and TYK2 tyrosine kinases. Experimental Hematology (1995) 23(9):1040-1048.

15. Budagian, V., Bulanova, E., Paus, R., and Bulfone-Paus, S.. IL-15/IL-15 receptor biology: A guided tour through an expanding universe. Cytokine Growth Factor Rev (2006) 17(4):259-280.

16. Budagian, V., Bulanova, E., Paus, R., and Bulfone-Paus, S.. Interleukin-15 Induces Rapid Tyrosine Phosphorylation of STAT6 and the Expression of Interleukin-4 in Mouse Mast Cells. Journal of Biological Chemistry (2000) 275(38):29331-29337.

17. Birgit, S.. Tyrosine kinase 2 (TYK2) in cytokine signaling and host immunity. Front Bio (2011)16(1):32143232 . 
18. Altan-Bonnet, G., and Mukherjee, R.. Cytokine-mediated communication: a quantitative appraisal of immune complexity. Nature Reviews Immunology (2019) 19(4):205-221.

19. Martínez-Barricarte, R., Markle,J.R., Ma, C.S., Deenick, E.K., Ramírez-Alejo, N., Mele, F., and Latorre, D.,et al. Human IFN- $\gamma$ immunity to mycobacteria is governed by both IL-12 and IL-23. Science immunology (2018) 3 (30):1-12.

20. HOLLAND, S.M.. STAT3 mutations in the hyper-IgE syndrome. N Engl J Med (2007) 357(16):16081619 .

21. Shimoda, K., Kato, K., Aoki, K., Matsuda, T., Miyamoto, A., and Shibamori, M., et al. Tyk2 Plays a Restricted Role in IFN $\alpha$ Signaling, Although It Is Required for IL-12-Mediated T Cell Function.Immunity (2000) 13(4):561-571.

22. Schwer D, T., Twigg, S., D Aschenbrenner, Manrique, S., and Uhlig, H. H.. A biallelic mutation in IL6ST encoding the GP130 co-receptor causes immunodeficiency and craniosynostosis. Journal of Experimental Medicine (2017) 214(9):2547-2562.

23. V Béziat, Tavernier, S. J., Chen, Y. H., Ma, C. S., and Puel, A.. Dominant-negative mutations in human IL6ST underlie hyper-IgE syndrome. Journal of Experimental Medicine (2020) 217(6):1-24.

24. Altin, J., Shen, C., and Liston, A.. Understanding the genetic regulation of IgE production. Blood Reviews (2010) 24(4-5):163-169.

25. Wu, L.C, Zarrin, A.A.. The production and regulation of IgE by the immune system. Nature Reviews Immunology (2014) 14(4):247-259.

26. Chatila, T. A., Blaeser, F., Ho, N., Lederman, H. M., and Bowcock, A. M.. JM2, encoding a fork head-related protein, is mutated in X-linked autoimmunity-allergic disregulation syndrome. The Journal of Clinical Investigation (2000) 106(12): R75-81.

\section{Figure Legends}

Figure 1. Identification of TYK2 mutations and TYK2 deficiency.

(A) Pedigrees of the TYK2-deficient families. Square and circle represent male and female respectively. The probands (P1, P2, P3, P4 and P5) in the five families are indicated by an arrow. (B) and (C) Schematic diagram of TYK2 gene and its 23 coding exons (B), TYK2 protein and its different domains (Ferm, SH2, Pseudokinase and Kinase) (C). The positions of the TYK2 mutations of the five patients are indicated by black arrows or vertical line.

(D) Confirmation by Sanger sequencing of the TYK2 gene in the patients and their parents.

(E-H) Western blot analysis of TYK2 expression in P1, P2, P3 and P5. Representative images from 2-3 independent experiments.

(I-L) qRT-PCR analysis of TYK2 transcription in P1, P2 and P3. Data are derived from 2-3 independent experiments.

\section{Figure 2. Analysis of cellular response to type I\&II IFNs in TYK2-deficient patients.}

(A-B) Total STAT1, STAT3 and tyrosine-phosphorylated STAT1(pSTAT1) and STAT 3 (pSTAT3) protein level were analyzed by western blot in P1, P2 and healthy control. The patients' PBMC were stimulated with IFN- $\alpha(100 \mathrm{ng} / \mu \mathrm{L})$, IFN- $\beta(500 \mathrm{IU} / \mathrm{mL})$ or IFN- $\gamma(100 \mathrm{ng} / \mu \mathrm{L})$ for $15 \mathrm{~min}$. Representative images from 2-3 independent experiments.

(C-D) Analysis of type I IFN-inducible gene induction in PBMCs from healthy control and P1 and P2. Cells were stimulated with IFN- $\alpha(100 \mathrm{ng} / \mu \mathrm{L})$, IFN $-\beta(500 \mathrm{IU} / \mathrm{mL})$ or without IFN for $4 \mathrm{~h}$. Data are derived from 2-3 independent experiments. 
Figure 3. Analysis of cellular response to IL-6 and IL-10 in TYK2- deficient patients.

(A, B and C) Total STAT3 and pSTAT3 protein level were analyzed by western blot in P1, P2, P3 and healthy control. The patients' PBMC were stimulated with IL-6 $(100 \mathrm{ng} / \mu \mathrm{L})$ or IL-10 $(100 \mathrm{ng} / \mu \mathrm{L})$ for 15 min. Representative images from 2-3 independent experiments.

(D) SOCS3 induction was analyzed by RT-qPCR after $6 \mathrm{~h}$ of treatment with $100 \mathrm{ng} / \mathrm{ml} \mathrm{IL-10,} \mathrm{in} \mathrm{PBMC}$ from healthy controls and P3. Data are derived from 2-3 independent experiments.

Figure 4. Analysis of cellular response to IL-12 and IL-23 of TYK2- deficient patients.

(A and B) Total STAT4 and tyrosine-phosphorylated STAT4 (pSTAT4) protein level were analyzed by western blot in P2, P3 and healthy control. The patients' PBMC were stimulated with IL-12 (100ng/ $\mu \mathrm{L})$ for $15 \mathrm{~min}$.

(C and D) Total STAT3 and pSTAT3 protein level were analyzed by western blot in P2, P3 and healthy control. The patients' PBMC were stimulated with IL-23 (100ng/ $\mu \mathrm{L})$ for $15 \mathrm{~min}$.

(E) Flow cytometry analysis showing intracellular IFN- $\gamma$ production in PBMCs after stimulation in the absence or presence of PMA $(50 \mathrm{ng} / \mathrm{ml})$ and Ionomycin $(500 \mathrm{ng} / \mathrm{ml})$ for $5 \mathrm{hrs}$ or BCG or BCG and $100 \mathrm{ng} / \mathrm{ml}$ IL-12 for 72 hrs. An anti-CD3 Ab was used to identify CD3 ${ }^{+} \mathrm{T}$ cells in P3 and healthy control.

Representative images from 2-3 independent experiments.

Figure 5. Characterization of T-cell and B-cell subsets in TYK2- deficient patients.

(A and B) Gating strategy for identifying Th1/2/17 (A), and Th1/2/17-like cells (B) by flow cytometry in healthy control and patients.

(C-F) Percentage of Th1/2/17, and Th1/2/17-like cells in P1, P2, P3 and healthy control (n=6).

(G- J) Gating strategy for identifying Tfh (G), Tfh expressing PD-1 (H), Tfr (I) and Treg (J) by flow cytometry in healthy control and patients.

(K, L and N) Percentage of Tfh, Tfh expressing PD-1, Tfr and Treg in P1, P2, P3 and healthy control (n=6). (M) Gating strategy for identifying $\operatorname{IgM}^{\text {hi }}(\mathrm{M})$, MZ-like (M) and Sm B cells (M) by flow cytometry in healthy control and patients.

$(\mathrm{O}$ and $\mathrm{P})$ The frequency of $\mathrm{B}$ cell subsets in P2 (O) and healthy control (n=5), P3 (P) and healthy control $(\mathrm{n}=6)$.

\section{SUPPLEMENTARY MATERIAL}

Supplementary Table 1. Primer sequences for Sanger sequencing of TYK2

Supplementary Table 2. Primer sequences used for qRT-PCR

Supplementary Table 3. Immunophenotyping of peripheral blood lymphocyte subsets

Supplementary Figure 1. Clinical manifestations of TYK2 deficient patients.

(A, B and C) stomatitis (A) accompanied with herpes (B) and BCG scar (C) in P1.

(D) BCG scar in P5.

\section{Supplementary Figure 2. Protein stability test of mutant TYK2.}

Cycloheximide Chase Assay for indicated mutants(A-E). Representative images from 2-3 independent experiments. 
Figure 1

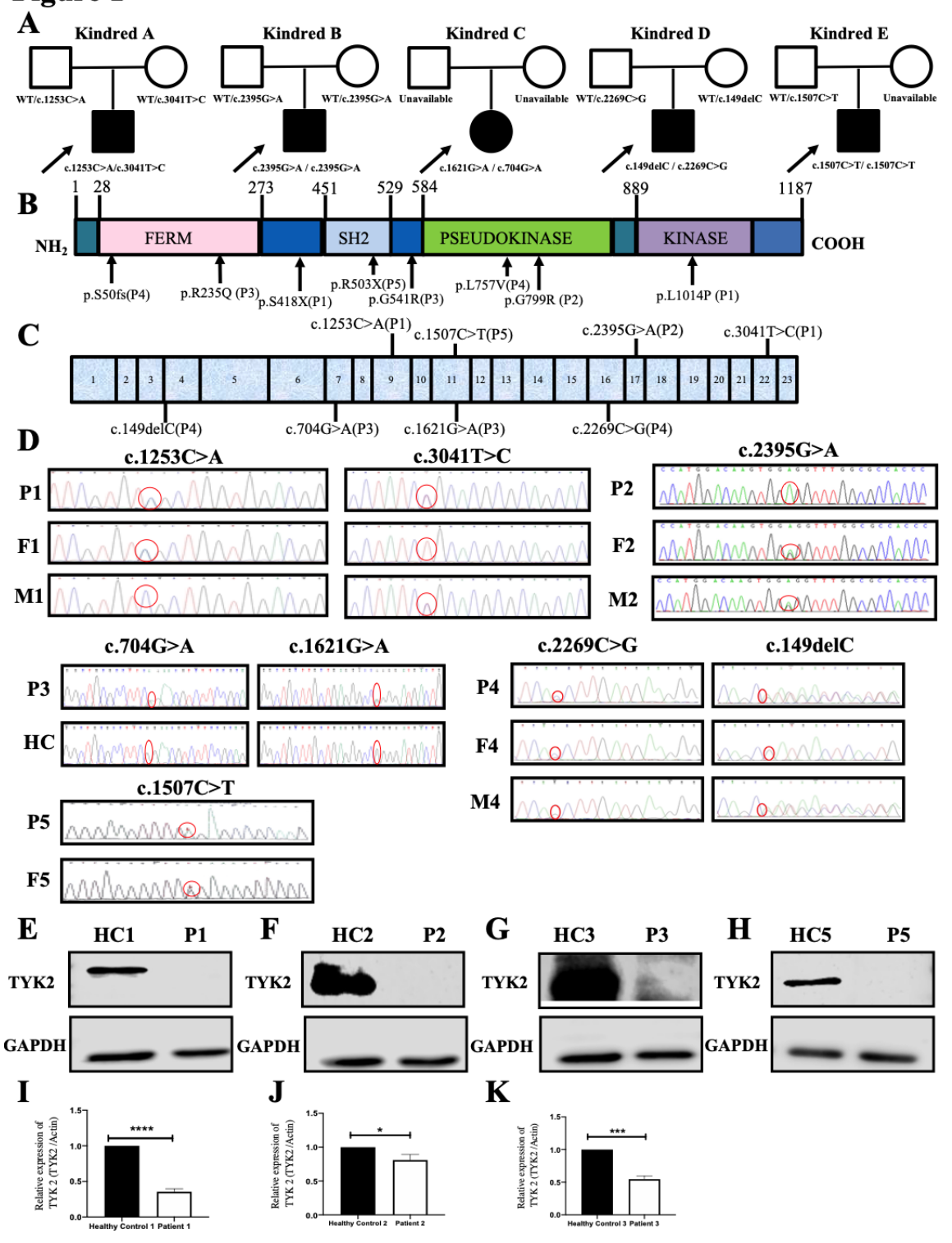


Figure 2

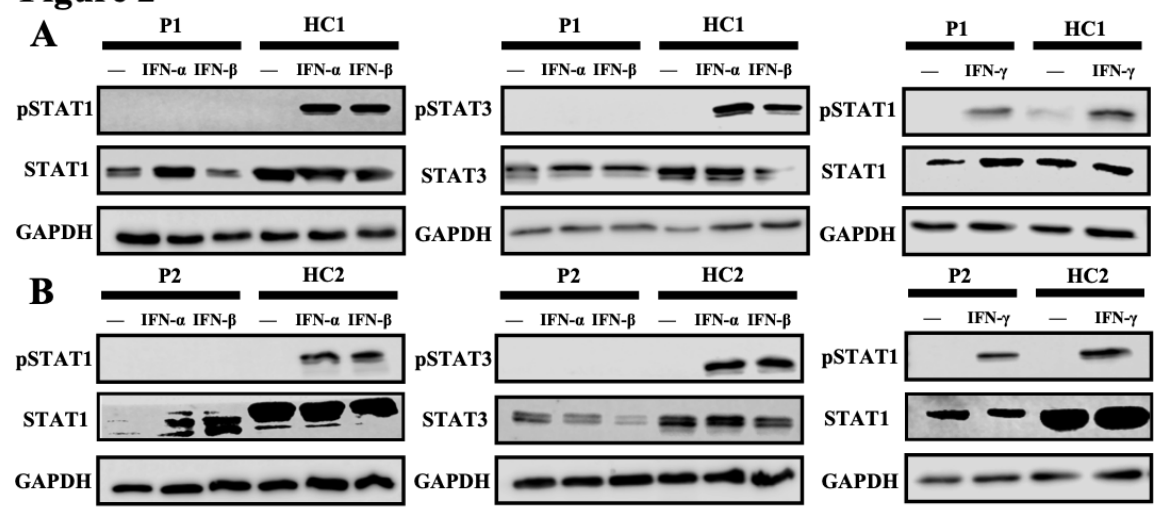

C
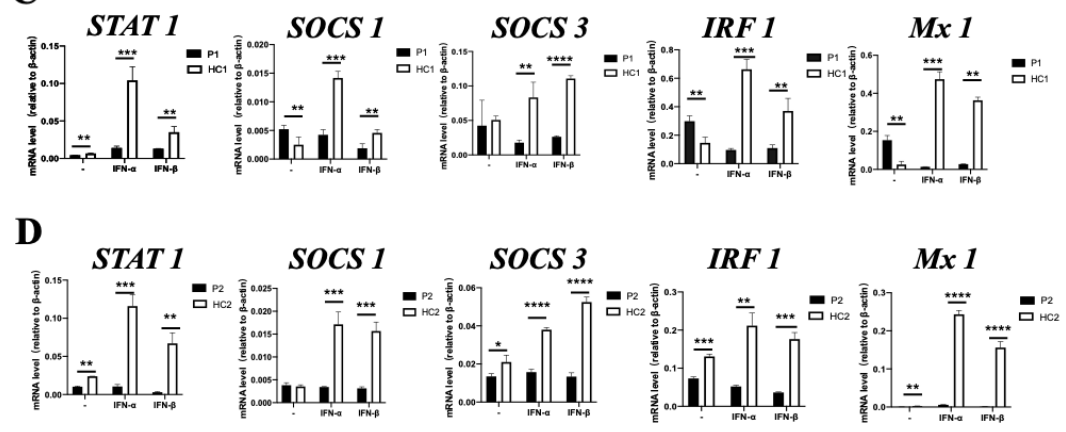

Figure 3
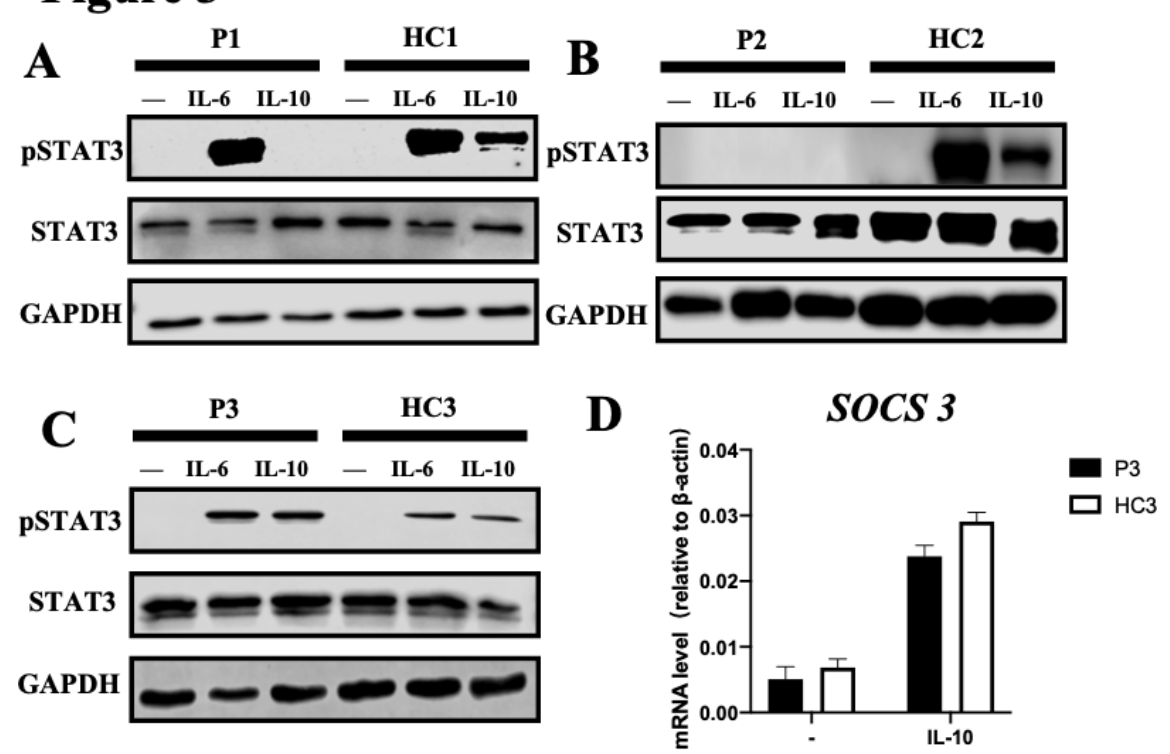
Figure 4

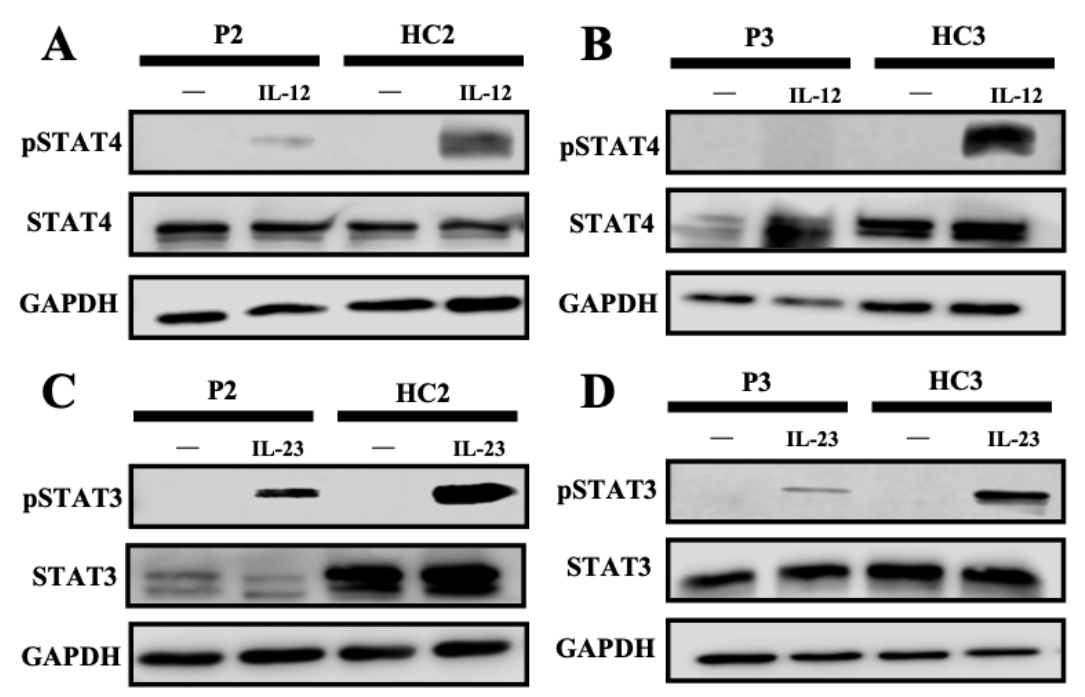

E
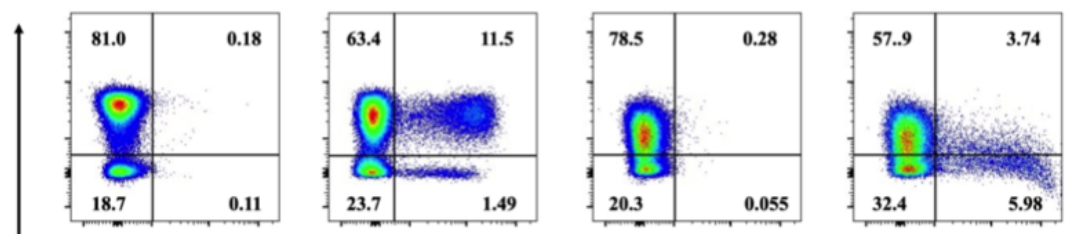

HC
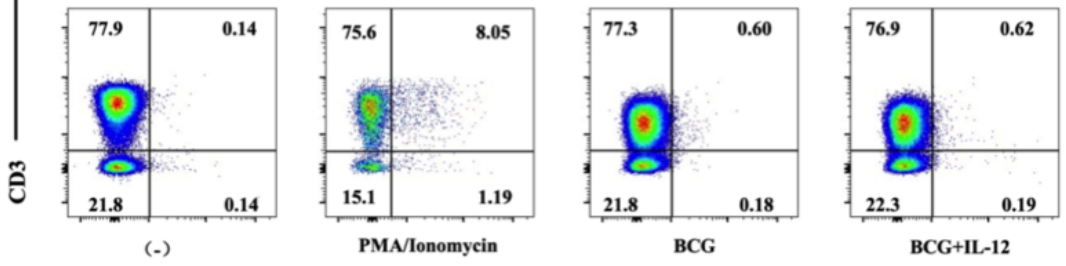

P3 IFN- $\gamma$ 
Figure 5
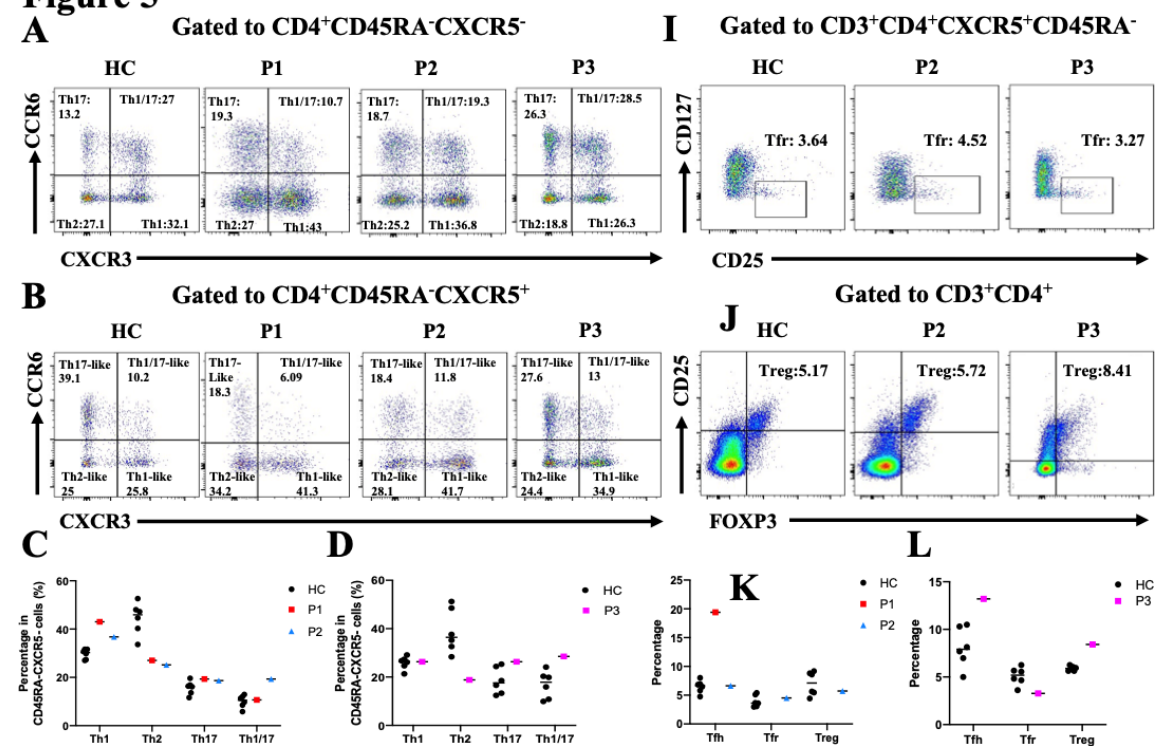

\section{$\mathbf{L}$}
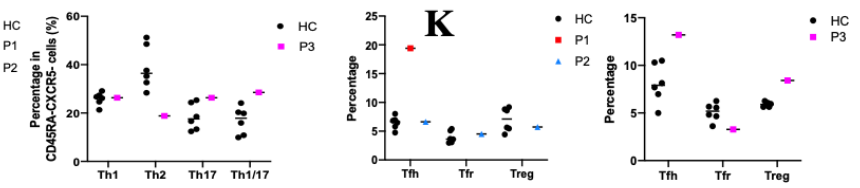

$\mathbf{E}$

$\mathbf{F}$

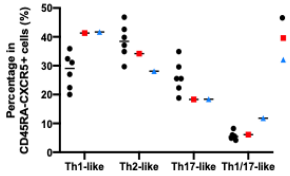

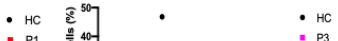

M

Gated to CD19 ${ }^{+}$
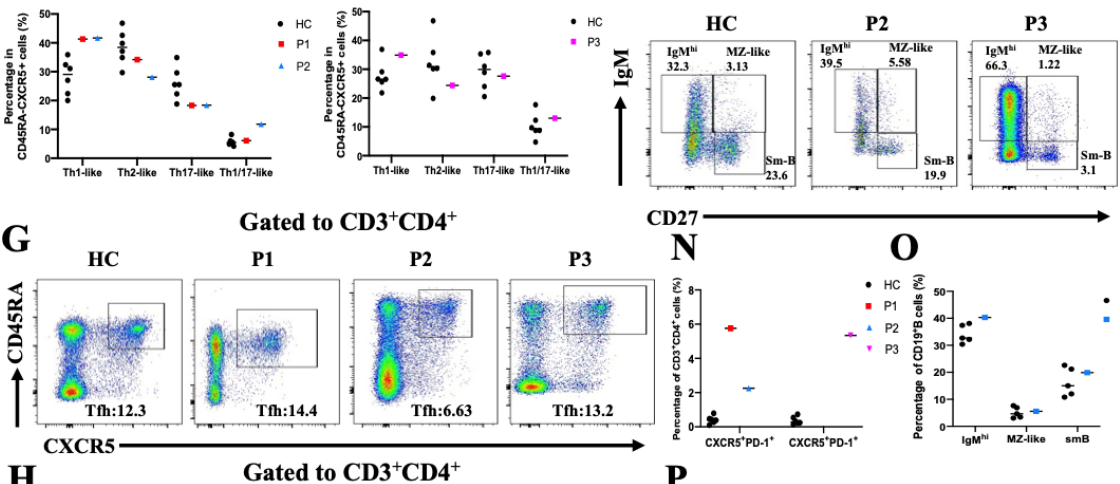

$\mathbf{N} \mathbf{O}$

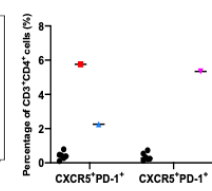

HC 疍

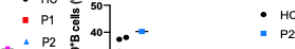

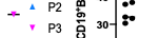

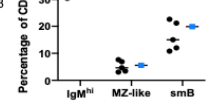

$\mathbf{H}$

P1 P2

$\mathbf{P}$

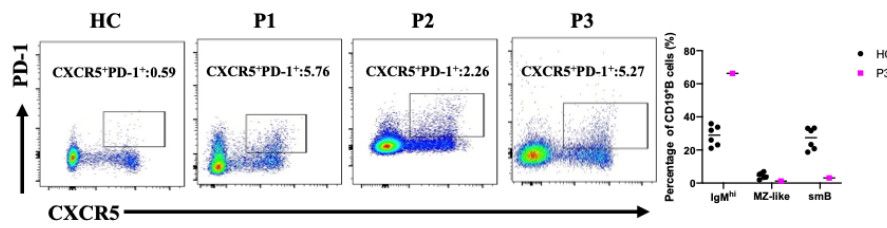

\section{Hosted file}

Table 1-for next.docx available at https://authorea.com/users/416396/articles/523963-novelmutations-of-tyk2-leading-to-divergent-clinical-phenotypes 